\title{
Atypical Location of Intracranial Germinoma: A Case Report
}

\author{
C. Piette $\cdot$ C. Hoyoux $\cdot$ O. Luckers $\cdot$ M. Tebache
}

Received: 19 July 2013 / Accepted: 27 November 2013 / Published online: 6 December 2013

C Springer-Verlag Berlin Heidelberg 2013

\section{Introduction}

Intracranial germinomas are rare and account for only 0.4 $3.4 \%$ of primary intracranial tumors. They predominantly develop around the onset of puberty and during the second and third decades of life and seem to be more frequent in Asia, where their incidence in children with a brain tumor reaches $2.1-9.4 \%$ [1].

These tumors usually develop in the midline structures, i.e., the pineal gland, hypothalamic region, and basal ganglia.

Herein, we report an atypical radiological presentation of intracranial germinoma, and we discuss the magnetic resonance imaging (MRI) characteristics and the diagnostic challenges of this entity.

\section{Case Report}

A previously fit and well 13-year-old boy of Russian origin presented with a 3-week history of vomiting and headaches and a 1-week history of diplopia. Physical examination dis-

C. Piette, MD, $\operatorname{PhD}(\triangle) \cdot$ C. Hoyoux, MD

University Department of Pediatrics, Hematology-Oncology, CHR Citadelle, Boulevard du Douzième de Ligne, 1 , 4000 Liège, Belgium

e-mail: caroline.piette@chrcitadelle.be

O. Luckers, MD

Department of Neurosurgery,

CHR Citadelle, Liège, Belgium

M. Tebache, MD

Department of Radiology,

CHR Citadelle, Liège, Belgium closed he was alert and oriented. Ophthalmologic examination showed a bilateral papilledema associated with bilateral sixth cranial nerve palsy. He had no other neurological deficit.

Cerebral MRI showed bilateral frontal periventricular lesions infiltrating the corpus callosum, the floor of the frontal horns, and the anterior wall of the left frontal horn (Fig. 1). These lesions were heterogeneous both in T1- and T2-weighted sequences and presented a slight diffusion restriction. Small cystic changes were visualized. Two other lesions were also visualized in the floor of the third ventricle and in the Sylvian aqueduct (Fig. 2), generating a supratentorial hydrocephalus associated with transependymal resorption signs. A complementary MRI centered on the hypothalamic-pituitary axis showed invasion of the posterior (mamillary) region of the hypothalamus and absence of invasion of the anterior (supraoptic) and middle (tuberal) regions of the hypothalamus, the pituitary gland, and the pineal gland. MRI of the spine showed no metastatic dissemination.

Biological results showed undetectable serum and cerebrospinal fluid (CSF) levels of alpha-fetoprotein and human chorionic gonadotropin. There were no tumoral cells in the CSF. Hormonal assessment showed decreased adrenocorticotropic hormone levels $(2.4 \mathrm{pg} / \mathrm{ml}$; normal values: $10-60 \mathrm{pg} / \mathrm{ml})$, reduced serum cortisol value $(0.4 \mu \mathrm{g} / \mathrm{dl}$; normal values: $6.2-19.4 \mu \mathrm{g} / \mathrm{dl}$ ), and low urinary cortisol excretion $(4 \mu \mathrm{g} / 24 \mathrm{~h}$; normal values: $10-85 \mu \mathrm{g} / 24 \mathrm{~h})$. There was no deficiency among the other pituitary hormones.

Endoscopic third ventriculostomy was performed and confirmed evidence of involvement of the floor of the third ventricle. Simultaneous biopsy sampling was obtained and revealed a pure germinoma (Fig. 3). 
Fig. 1 Coronal (a) and axial (b) postcontrast T1-weighted slices showing bilateral frontal periventricular lesion infiltrating the genu of corpus callosum, the floor and the anterior wall of the left frontal horn, and the tumorassociated vasogenic edema, with supratentorial hydrocephalus associated with transependymal cerebrospinal fluid resorption. Coronal T2-weighted slice (c) showing several small cystic changes (arrow). Axial apparent diffusion coefficient map from diffusion sequence (d) showing slight diffusion restriction of the paraventricular lesion (arrow)


Computed tomography scan of the chest, abdomen, and pelvis and ultrasonography of the scrotum did not show any primary or metastatic lesion.

The patient was treated with radiotherapy alone. Radiation therapy consisted of $24 \mathrm{~Gy}$ to the craniospinal axis, followed by a 16 Gy boost to the tumor bed and the intracranial metastasis, thereby increasing the tumor bed and metastasis dose to 40 Gy. The MRI performed 1 month after the end of radiotherapy showed complete resolution, and the patient remained stable without evidence of recurrent disease 14 months after completion of radiotherapy.

\section{Discussion}

The main distinguishing radiological features of pure germinomas relate to their typical sites of involvement. They are indeed most often located in the midline structures, i.e., the pineal gland, hypothalamic region, and basal ganglia. Approximately $30 \%$ of them are bifocal, involving both the suprasellar and pineal region.

Other sites have rarely been described, including the corpus callosum [2], the corona radiata [3], and the frontal lobe [4]. The incidence of metastatic intracranial germinomas is not well established and varies largely among series. In a series of 23 patients [5], 34\% were found to have CSF seed- 
Fig. 2 Postcontrast T1-weighted axial (a) and midline sagittal (b) slices showing the tumor seeding into the Sylvian aqueduct responsible for the supratentorial hydrocephalus (arrows), the infiltration of corpus callosum, the tumor lining the anterior columns of the fornix, and the dissemination throughout the ventricular system. The irregular enhancement in the pineal region (b) appeared unchanged post-treatment (data not shown), indicating that it cannot be considered as tumoral involvement of the pineal gland


ing. Nevertheless, this percentage is probably overestimated because of the exclusion of 19 patients without histologically proven germinomas, which were probably mostly typical and localized ones. In another small series of eight histologically verified germinomas [6], one (12.5\%) was metastatic. Finally, Lee et al. [7] reported 30 histologically confirmed germinomas, among which there was no metastatic case. Most commonly, germinoma spread to the brain ventricle. Meningeal disseminations with subdural lesions are rarely seen [8]. In several series, a longer interval between the first symptoms and the diagnosis seemed to be associated with a higher incidence of metastatic germinomas.

When suspected, the diagnosis of intracranial germ cell tumor should lead to a complete diagnostic staging, including brain and spinal MRI, CSF cytology, and tumor markers (alpha-fetoprotein and human chorionic gonadotropin) measurement in both serum and CSF. Indeed, it has been shown that the incompleteness of diagnostic staging correlates to the occurrence of relapses, with relapses occurring in $7 \%$ of completely worked-up patients and in $24 \%$ of incompletely worked-up patients, respectively [9].

One of the particularities of this case is the existence of cortisol deficiency without radiological evidence of involvement of the anterior and middle regions of the hypothalamus and of the pituitary gland, suggesting possible delayed MRI findings in these areas. In a single-institution review, 2 patients out of 10 with germinoma presented diabetes insipidus without MRI evidence of neoplastic involvement of the third ventricle floor, but with endoscopic evidence of neoplastic involvement of this region [10]. These observa- tions illustrate the importance of a comprehensive clinical and biological assessment of the hypothalamic-pituitary axis in complex cases.

In the presented case, most parts of the lesions were intraparenchymal, and there was no involvement of the pituitary gland and the pineal gland, making the radiological findings nonspecific. The differential diagnosis of periventricular tumors in children and adolescents comprises ependymoma, lymphoma, supratentorial primitive neuroectodermal tumor (PNET), and glioblastoma. Typically, ependymoma shows heterogeneous enhancement with cystic areas; lymphoma in immunocompetent patients presents as a homogeneously enhancing parenchymal mass; supratentorial PNET is heterogeneous with moderate gadolinium enhancement; and glioblastomas often contain areas of necrosis. Nevertheless, these criteria are not specific. Furthermore, advanced imaging such as diffusion and spectroscopy does not reliably differentiate these different tumor types.

In conclusion, although this kind of radiological presentation is uncommon, germinoma should also be included in the differential diagnosis of infiltrative parenchymal tumors invading the ventricular system, especially among adolescents and young adults, even if midline structures seem to be spared on classical MRI. This is of importance to guide the diagnosis approach and to carry out a complete diagnostic staging.

Acknowledgments The authors would like to thank Dr. Alex Michotte for providing the photomicrographs. 

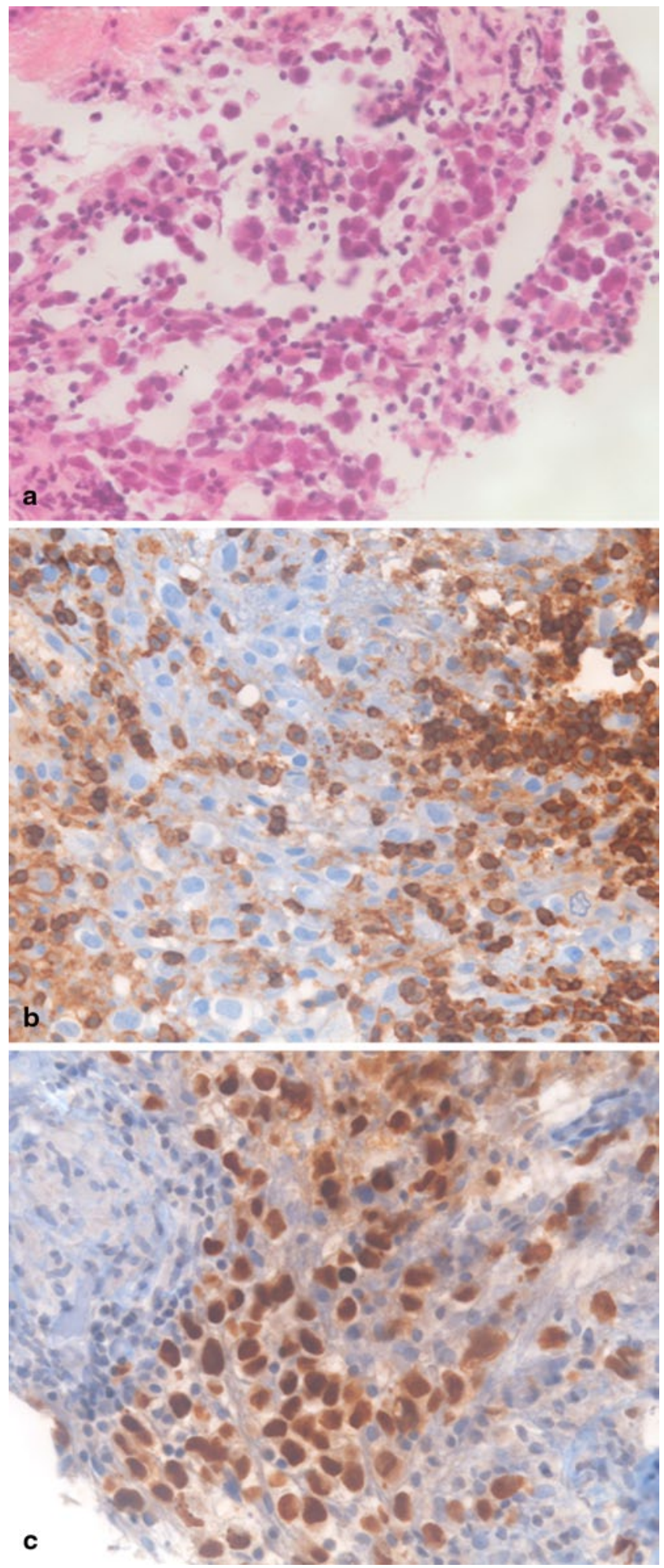

Fig. 3 Photomicrographs of histological findings (original magnification $\times 400$ ). Hematoxylin and eosin stain (a) showing two-cell pattern of germinoma with small lymphoid elements and large poorly differentiated cells with abundant cytoplasm and round nuclei. Immunohistochemical stainings of CD45 (b) revealing positive lymphocytes and of OCT4 (c) revealing positive tumor cell nuclei
Conflict of Interest On behalf of all authors, the corresponding author states that there is no conflict of interest.

\section{References}

1. Hoffman HJ, Otsubo H, Hendrick EB, Humphreys RP, Drake JM, Becker LE, et al. Intracranial germ-cell tumors in children. J Neurosurg. 1991;74(4):545-51. doi:10.3171/jns.1991.74.4.0545.

2. Utsuki S, Oka H, Tanizaki Y, Kondo K, Fujii K. Radiological features of germinoma arising from atypical locations. Neurol Med Chir. 2005;45(5):268-71.

3. Okuno S, Hisanaga M, Chitoku S, Sakaki T, Tsunoda S. [Germinoma with granulomatous reaction arising from the corona radiata; case report and review of articles]. No Shinkei Geka. 1992;20(7):775-80.

4. Odake G, Horikawa Y, Yoshino E, Hirakawa K. [Bifrontal cystic tumor - a form of suprasellar germinoma (author's transl)]. No Shinkei Geka. 1980;8(4):383-8.

5. Moon WK, Chang KH, Han MH, Kim IO. Intracranial germinomas: correlation of imaging findings with tumor response to radiation therapy. AJR Am J Roentgenol. 1999;172(3):713-6. doi:10.2214/ajr.172.3.10063866.

6. Shokry A, Janzer RC, Von Hochstetter AR, Yasargil MG, Hedinger C. Primary intracranial germ-cell tumors. A clinicopathological study of 14 cases. J Neurosurg. 1985;62(6):826-30. doi:10.3171/ jns.1985.62.6.0826.

7. Lee D, Suh YL. Histologically confirmed intracranial germ cell tumors; an analysis of 62 patients in a single institute. Virchows Arch. 2010;457(3):347-57. doi:10.1007/s00428-010-0951-3.

8. Takeshima Y, Yamada S, Motoyama Y, Park YS, Nakase H. An unusual case of primary central nervous system germinoma with meningeal dissemination. Childs Nerv Syst. 2012;28(12):2173-6. doi:10.1007/s00381-012-1901-y.

9. Calaminus G, Alapetite C, Bamberg M, Baranzelli MC, Frappaz $\mathrm{D}$, Garré ML, et al. Malignant CNS germ cell tumours: interim analysis after 5 years of SIOP CNS GCT 96. Med Pediatr Oncol. 2002;39:227.

10. Wellons JC, 3rd, Reddy AT, Tubbs RS, Abdullatif H, Oakes WJ, Blount JP, et al. Neuroendoscopic findings in patients with intracranial germinomas correlating with diabetes insipidus. J Neurosurg. 2004;100(5 Suppl Pediatrics):430-6. doi:10.3171/ ped.2004.100.5.0430. 